Case Report:

\title{
Concomitant Primary Cerebral Lymphoma and Meningioma
}

\author{
Ehsan Mohammadhosseini ${ }^{1^{*}}$, Bahram Aminmansour ${ }^{1}$, Susan Andalibi² ${ }^{2}$ Maryam Derakhshan ${ }^{3}$, Fataneh Farokhpour
}

1. MD. Department of Neurosurgery, AL-Zahra Hospital, Isfahan University of Medical Sciences, Isfahan, Iran

2.MD. Department of Radio Oncology, Omid Hospital, Isfahan University of Medical Sciences, Isfahan, Iran

3. MD. Department of Pathology, AL-Zahra Hospital, Isfahan University of Medical Sciences, Isfahan, Iran

$\begin{aligned} & \text { Use your device to scan } \\ & \text { and read the article online }\end{aligned}$
Lymphoma and Meningioma. Iran J Neurosurg. 2019; 5(1):43-50. http://dx.doi.org/10.32598/irjns.5.1.43
dcit": http://dx.doi.org/10.32598/irjns.5.1.43

(c) 0

Article info:

Received: 25 Jul 2018

Accepted: 12 Nov 2018

Available Online: 01 Jan 2019

Keywords:

Concomitant Cerebral,

Lymphoma,

Meningioma

\section{A B STRACT}

Background and Importance : Primary Central Nervous System Lymphoma (PCNSL) is a rare cancer confined to the brain, spinal cord, leptomeninges, and eyes. The origin of this type of lymphoma is often type B lymphocytes, and because the Central Nervous System (CNS) has no lymph nodes or lymphatic vessels, the cause of PCNSL is still unclear. But its source appears to be from lymphocytes located in the CNS. This type of lymphoma has been reported in the context of congenital or acquired immune deficiency such as Wiskott Aldrich syndrome, kidney transplantation, and, in particular, AIDS. Frontal lobes are the most common sites of involvement in CNS.

Case Presentation: The patient was a 55-year-old man who had been referred to the Emergency Department of Al-Zahra Hospital, Isfahan, Iran due to recurrent headaches, nausea, and vomiting since 6 months ago.

Conclusion: Meningiomas are often benign and slowly growing tumors that originate from the arachnoid cap cell of meninges. Due to the rarity of concomitance of primary cerebral lymphoma and meningioma, there is no possibility of epidemiological studies in these patients.

\section{* Corresponding Author:}

Ehsan Mohammadhosseini, MD.

Address: MD, Department of Neurosurgery, AL-Zahra Hospital, Isfahan University of Medical Sciences, Isfahan, Iran

Tel: +98 (31) 36692174

E-mail: ehsan_m_h76@yahoo.com 


\section{Highlights}

- Concomitant primary cerebral lymphoma and meningioma is a rare type of CNS cancer with no predictable pattern of recurrence, therefore, any information on this matter may help scientists in the fields of neurosurgery and epidemiology.

- As the early diagnosis could increase the five year survival of newly diagnosed patients by $70 \%$, symptoms which vary depending on the site of the tumor, should be taken under serious consideration.

- If suspected, detailed paraclinical examinations including CT scan, MRI, blood biomarkers, and histopathological studies are recommended.

\section{Plain Language Summary}

Primary cerebral ly mphoma is a rare type of brain tumor arising from type Blymphocytes. Although it is very rare, itcan alsobesuperimposed toothertypesofbraintumors, likemeningiomas.Inthiscaseitishardtocometoanaccuratediagnosis.

\section{Background and Importance}

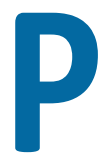

rimary Central Nervous System Lymphoma (PCNSL) is a rare cancer confined to the brain, spinal cord, leptomeninges and eyes. The origin of this type of lymphoma is often type B lymphocytes, and because the Central Nervous System (CNS) has no lymph nodes or lymphatic vessels, the cause of PCNSL is still uncertain [1]. However, its source appears to be from lymphocytes located in the CNS [2]. This type of lymphoma has been reported in the context of congenital or acquired immune deficiency such as Wiskott Aldrich syndrome, kidney transplantation, and in particular AIDS [3]. The frontal lobes are the most common site of involvement in the CNS. Although, personality disorders and altered levels of consciousness are the hallmarks of the disease, the rate of seizure is lower compared to other types of brain tumors $[3,4]$.

PCNSL grows rapidly, with clinical symptoms appearing a few weeks to several months before the diagnosis. If not treated, this disease can lead to death within one to three years. However, some studies have shown that if treated, $70 \%$ of the affected people can survive up to 5 years after the diagnosis [3]. Meningiomas are often benign and slowly growing tumors that originate from the arachnoid cap cell of the meninges [4, 5]. Meningioma is a highly prevalent tumor in the CNS, but the primary brain lymphoma is a rare, non-Hodgkin's subtype tumor. Because of the heterogeneity and unpredictability of these two types of tumors, their concurrency is rare in one patient [6].

\section{Case Presentation}

The patient was a 55-year-old man who had been referred to the Emergency Department of Al-Zahra Hospital, Isfahan, Iran due to recurrent headaches, nausea, and vomiting since 6 months ago. In his physical examination, cranial nerves were intact, with a Glasgow Coma Scale of $15 / 15$. Cerebellar tests were abnormal with observed vertigo and ataxia. Both pupils were normally sized, symmetric, and reactive to light. Sensory and motor function of the four extremities were normal. There were no reports of seizure, diplopia and aphasia. The patient was diabetic and treated with metformin and aspirin. He had no history of other malignancies or positive family history.

Magnetic Resonance Imaging (MRI) was performed for better evaluation. In the T1 weighted view, a welldefined hypo signal mass was seen in the cerebellum with the compression on the fourth ventricle and shift of the fourth ventricle to the left side (Figure $1 \mathrm{~A}$ ). In the T2 weighted, cerebellar mass was heterogeneous and a hyper signal was seen with a hypo signal center (Figure 1B). After intravenous administration of Gadolinium, moderate and heterogeneous enhancement of the cerebellar lesion was seen (Figure 1C). In DiffusionWeighted MRI (DW-MRI) and apparent diffusion coefficient $(A D C)$, the mass showed diffusion restriction (Figure 1D-1E).

A hypo signal, extra axial mass was also observed in the right frontal lobe in T1 weighted images (Figure 1F). The frontal lesion was homogeneous and a hyper sig- 
A

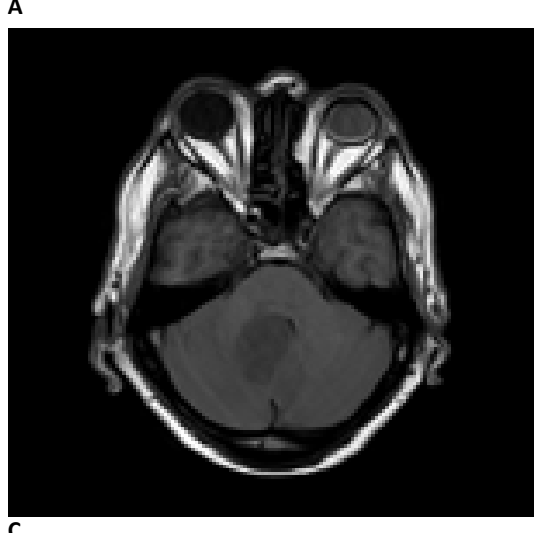

C
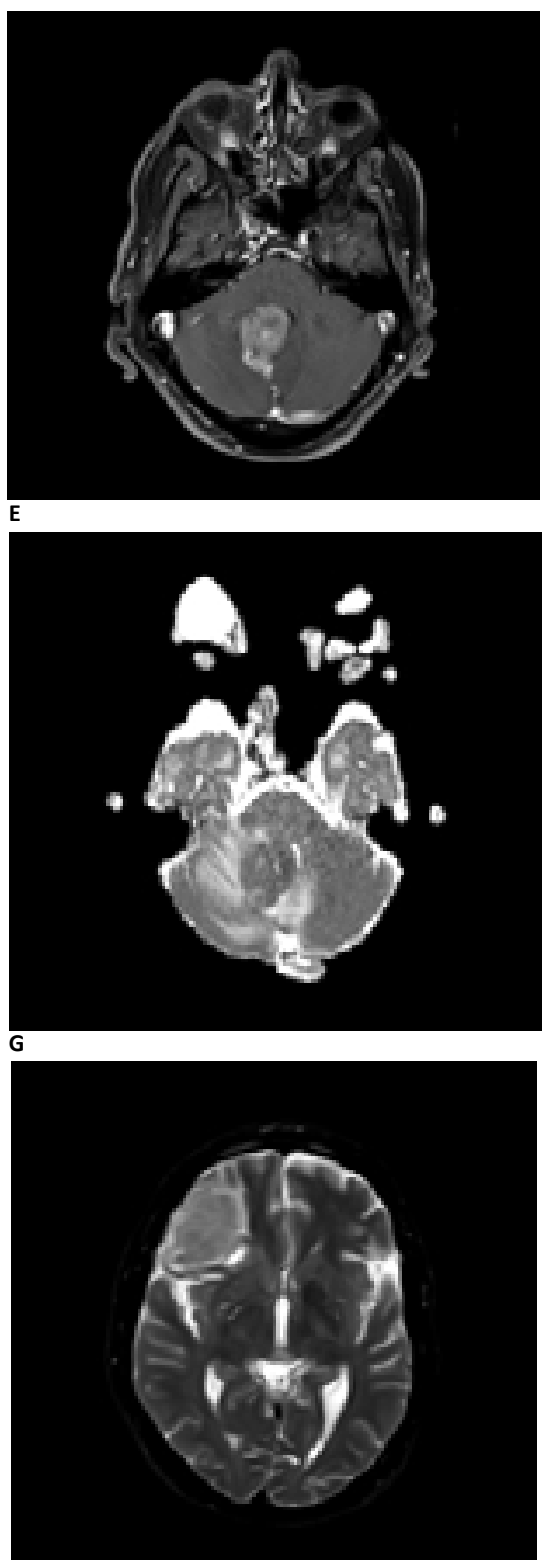

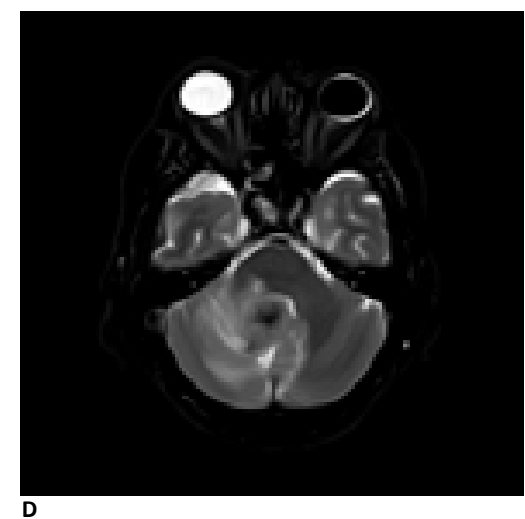

D
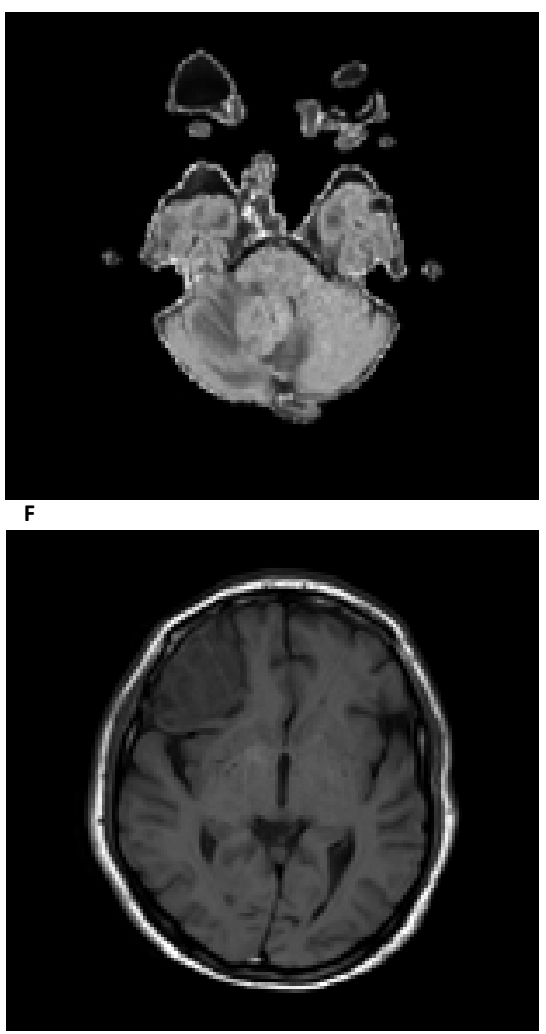

H

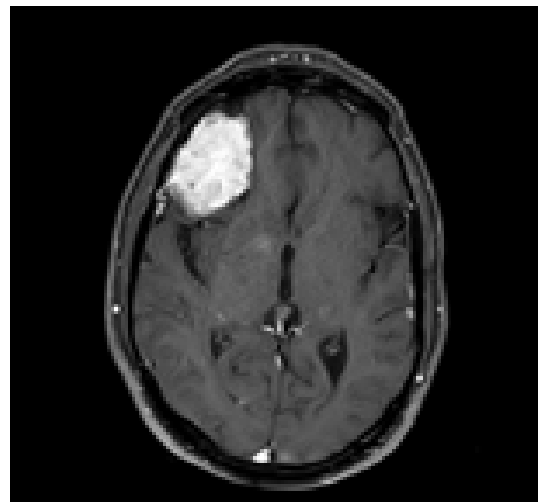

Figure 1. Magnetic resonance imaging of a patient with cerebellar lymphoma and frontal meningioma

A: A hypo signal mass in the cerebellum; B: Heterogeneous and hyper signal with hypo signal center; C: Moderate and heterogeneous enhancement of cerebellar lesion; D and E: Diffusion restriction of mass in DWI and ADC; F and G: An extra axial mass, hypo signal in T1 and hyper signal in $\mathrm{T} 2$, respectively; $\mathrm{H}$ : Intense homogeneous contrast enhancement with dural tail sign in frontal mass. 


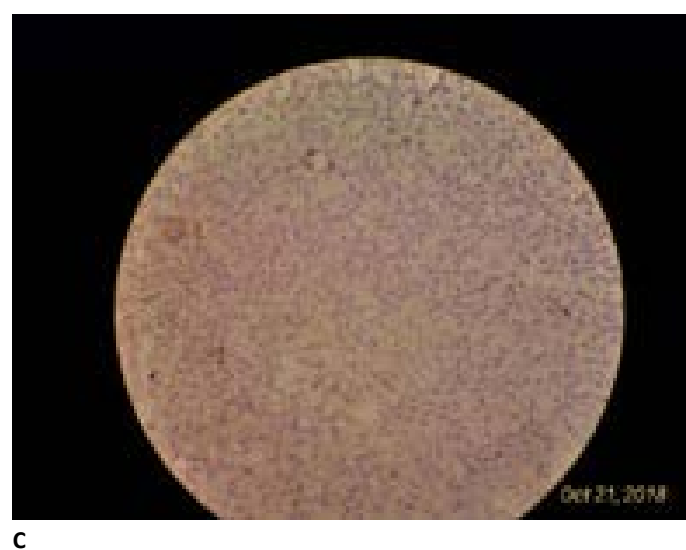

C

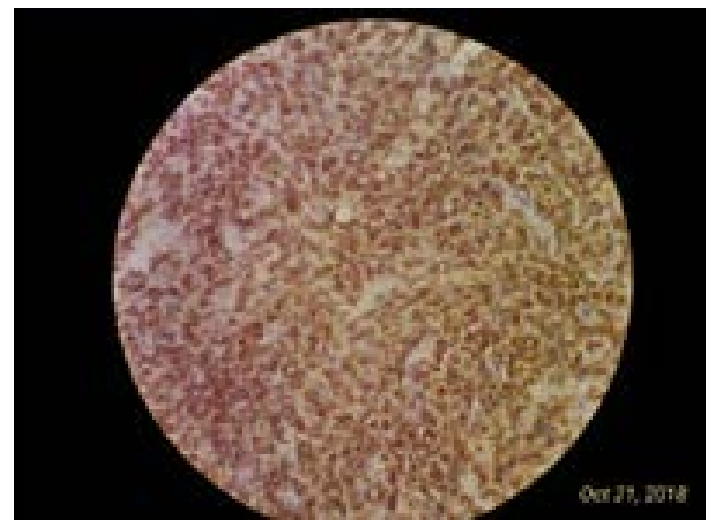

$\mathbf{E}$

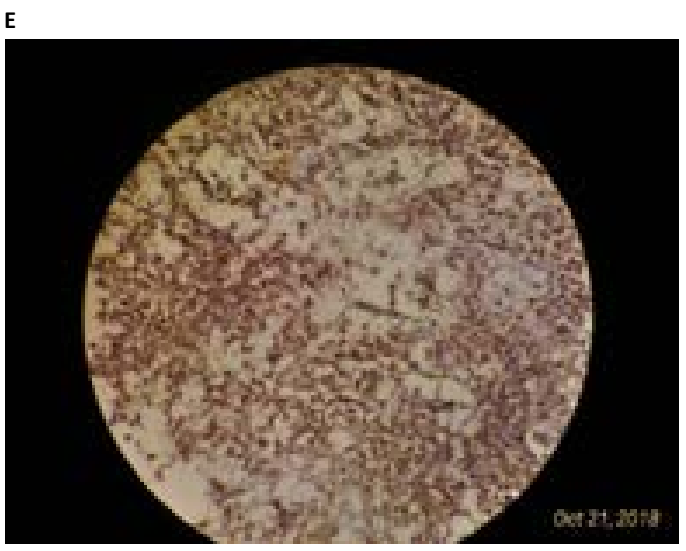

Figure 2. Histopathology of patient with cerebral lymphoma with IHC staining

A. Negative for EMA; B. Negative for GFAP; C. Positive for CD20; D. Negative for CD3; E. positive for LCA nal was observed in T2 weighted (Figure 1G). However, in the frontal lesion, an intense homogeneous contrast enhancement and a dural tail sign, mimicking meningioma, was observed (Figure $1 \mathrm{H}$ ).

The patient was scheduled for surgery. Surgical resection was done in two stages at intervals of one week. In the first stage, in prone position, sub occipital craniotomy was done. Using the Telovelar approach, a biopsy of cerebellar mass was done with a frozen section prepared. Considering the sampling response that
B

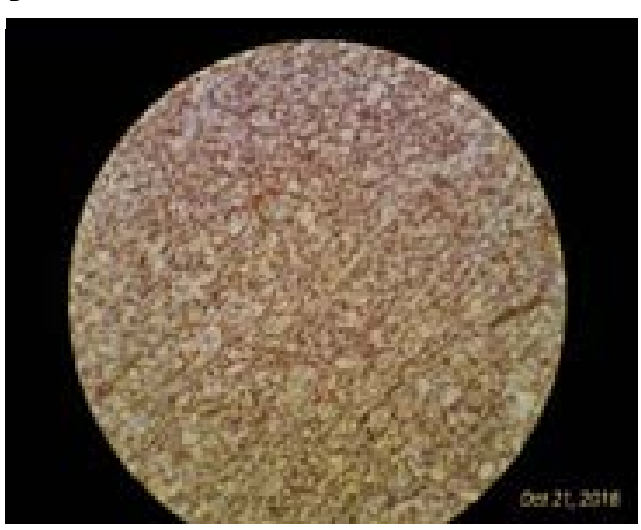

D

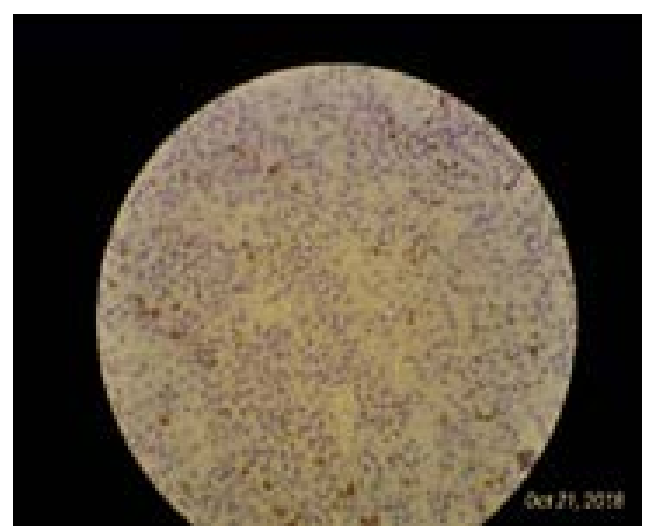

Shet, and

reported lymphoma, the surgery was terminated. The patient was admitted to the neurosurgical Intensity Care Unit (ICU).

Following the surgery, a part of the tumor was excised and sent to pathology for sampling. A microscopic review showed proliferation of medium to large lymphoid cells with hyperchromatic nuclei and high N/C ratio. In Immunohistochemistry (IHC) examination, GFAP, CK, EMA, CD3, and CD10 markers were negative and the results of S100, LCA, CD20 and KI 67 were posi- 


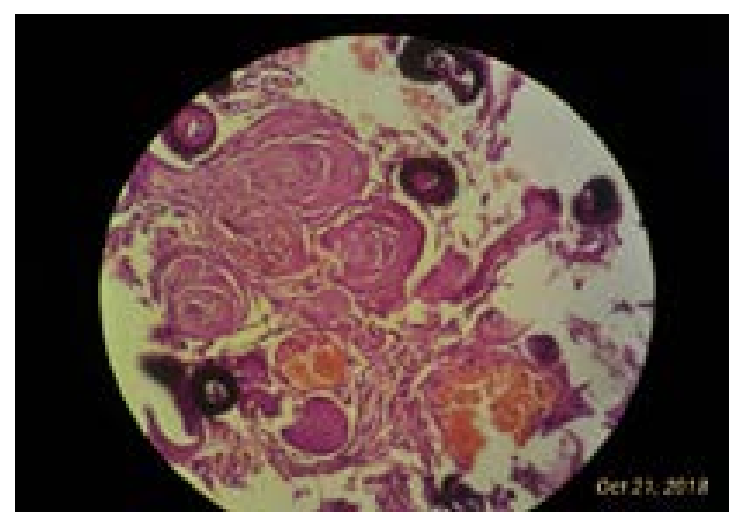

Figure 3. Histopathology of patient with angiomatous meningioma tive. The patient's pathological diagnosis was large B cell lymphoma (Figure 2).

In the second stage of surgery, in supine position, a right side frontotemporal craniotomy was done. With the dura opened in a Cshaped fashion, an extra axial mass with dural attachment was totally resected.

Examination of the histopathology of this lesion showed proliferation of meningothelial cells with the eosinophilic cytoplasm, intranuclear cytoplasmic pseudoinclusions, and psammoma body arranged in the sheet of the cell, along with multiple small and large vessels, which determined the diagnosis of angiomatous meningioma (Figure 3).

After surgery, the patient was transferred to the neurosurgical ICU and the next day was transferred to the neurosurgery ward. In post-surgical examinations, no new neurological deficits were detected, and the patient was discharged with a Glasgow Coma Scale of 15/15.

Because of the pathological result that reported the cerebellar mass as a large B cell lymphoma, the patient was examined for bone marrow specimens, which along with CT scans of the chest, abdomen, and
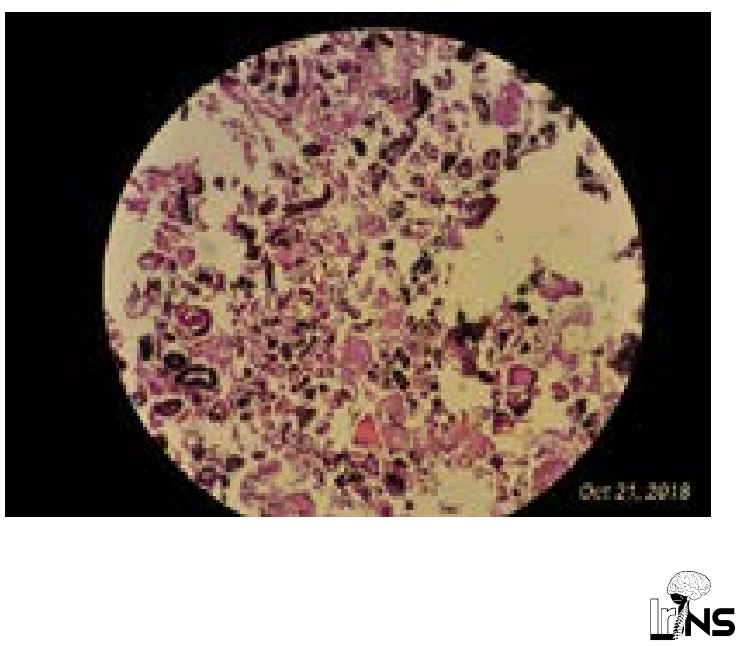

pelvis, were reported normal. Also, viral infections of hepatitis B, C, and HIV were also reported negatively. Finally, the patient was referred to the oncology service for continued treatment.

\section{Discussion}

The concomitancy of the primary cerebral lymphoma and meningioma is very rare, and the reported cases are very limited worldwide. In a case report presented by İldan et al. in 1995, the concurrence of primary lymphoma and meningioma was reported in a 38-year-old woman with a headache complaint and papilla edema, which was reported in MRI and pathology studies of the patient [6]. Another case of the coexistence of primary lymphoma and meningioma was reported by Erdem et al. in 2012 from Turkey. The patient was a 71-year-old woman with vertigo, imbalance, and headache (Table 1) [7].

In 2011, Amirjamshidi et al. reported a case of a 16-year-old boy presenting different types of brain tumors in two ventricular chambers concomitantly. This boy had a medium-sized colloid cyst of the third ventricle and a large fibrillary astrocytoma fungating from the brainstem into the floor of the fourth ventricle. The

Table 1. Characteristics of the concomitancy of the primary cerebral lymphoma and meningioma in other reports

\begin{tabular}{ccccc}
\hline Author & Year & Patient Age & Patient Sex & Compliant \\
\hline Lidan & 1995 & 38 & female & Headache, papilla edema \\
Erdem & 2012 & 71 & female & Vertigo, imbalance and headache \\
\hline
\end{tabular}


lesions were successfully excised in two separate surgeries. Radiotherapy was used as the adjuvant mode of therapy. There was no sign of tumor recurrence after 16 months of follow-up. Clinical awareness and diagnosis of such tumor combinations are very crucial, since they require certain therapeutic strategies that consider the aggressiveness of all malignancies [8].

The PCNSL is a rare tumor with a $4 \%$ prevalence among central nervous system tumors, and on the other hand, the concurrence of this tumor with other brain tumors is rare [9]. Therefore, it is very difficult to diagnose the coincidence of primary cerebral lymphoma with meningioma in conventional studies. If there is a suspicion of the presence of PCNLS and other types of tumors in a cerebral mass, detailed Para clinical examinations, such as CT scan, MRI and histopathologic studies, are required, especially in cases where there is no history of a previous tumor, the use of carcinogens, or previous radiotherapy $[10,11]$.

Concerning the incidence of primary cerebral lymphoma and meningioma, several hypotheses have been suggested including immunodeficiency disorders, such as the risk of AIDS; Moreover, the prevalence of PCNSL was reported in $20 \%$ of patients with AIDS [12, 13]. On the other hand, the presence of some features, including the positive genetic markers (also found in the CD20 in our patient), should also be considered [14].

\section{Conclusion}

Due to the rarity of concomitance of primary cerebral lymphoma and meningioma, there is no possibility of epidemiological studies in these patients. Because of an increase in the prevalence of risk factors that contribute to the development of brain tumors, including immunodeficiency diseases, exposure to radiation, and other factors whose harmful effects are not yet known to mankind, it is necessary that in the face of brain tumors, the probability of the coexistence of the primary tumor with other tumors is considered and clinical and para clinical examinations including histopathology, radiology, and laboratory procedures are carried out with precision before treatment.

\section{Ethical Considerations}

Compliance with ethical guidelines

All ethical principles were considered in this article.
Funding

This research did not receive any specific grant from funding agencies in the public, commercial, or not-forprofit sectors.

Authors contributions

All authors contributed in preparing this article.

Conflict of interest

The authors declared no conflict of interest.

\section{References:}

[1] Lukas RV, Stupp R, Gondi V, Raizer JJ. Primary central nervous system lymphoma-PART 1: Epidemiology, diagnosis, staging, and prognosis. Oncology. 2018; 32(1):17-22. [PMID]

[2] Cantwell L, Ramakrishnan A, Shaughnessy PJ, Bachier CR, Selby G, Bhushan V, et al. Autologous stem cell transplant in patients with primary central nervous system lymphoma: A multicenter analysis from the Sarah cannon blood cancer network. Biology of Blood and Marrow Transplantation. 2018, 24(3):S259-60. [DOI:10.1016/j.bbmt.2017.12.238]

[3] Li V, Levine AB, Gooderham PA, Yip S, Chew J. Case of primary central nervous system lymphoma arising at site of remote herpes encephalitis. World Neurosurgery. 2018; 113:217-22. [DOI:10.1016/j.wneu.2018.01.143] [PMID]

[4] Baldi I, Engelhardt J, Bonnet C, Bauchet L, Berteaud E Grüber A, et al. Epidemiology of meningiomas. Neurochirurgie. 2018; 64(1):5-14. [DOI:10.1016/j.neuchi.2014.05.006] [PMID]

[5] Zouaoui S, Darlix A, Rigau V, Mathieu-Daudé H, Bauchet F, Bessaoud F, Fabbro-Peray P, et al. Descriptive epidemiology of 13,038 newly diagnosed and histologically confirmed meningiomas in France: 2006-2010. Neurochirurgie. 2018; 64(1):15 21. [DOI:10.1016/j.neuchi.2014.11.013] [PMID]

[6] İldan F, Bağdatoğlu H, Boyar B, Haciyakupoğlu S, Gönlüşen G, Tunali N. Combined occurrence of primary cerebra lymphoma and meningioma. Neurosurgical Review. 1995; 18(1):45-8. [DOI:10.1007/BF00416477] [PMID]

[7] Erdem H, Uzunlar KA, Yildirim U, SAV A, Dosoglu M. Collision tumor of meningioma and non Hodgkin malignant lymphoma of cerebellum. Brain Disorders \& Therapy. 2012 1(1):1000103. [DOI:10.4172/2168-975X.1000103]

[8] Amirjamshidi A, Shams Amiri R, Alimohammadi M, Abbasioun K. Concomitant intraventricular colloid cyst and low-grade astrocytoma of the brainstem in a 16-year-old boy, case report. Journal of Neurosurgery. 2011; 8(4):342-5. [DOI:10.3171/2011.7.PEDS11179] [PMID]

[9] Hoffman S, Propp JM, McCarthy BJ. Temporal trends in incidence of primary brain tumors in the United States, 
1985-1999. Neuro-Oncology. 2006; 8(1):27-37. [DOI:10.1215/ S1522851705000323] [PMID] [PMCID]

[10] Aldape K, Simmons ML, Davis RL, MiikeR, WienckeJ, Barger $\mathrm{G}$, et al. Discrepancies in diagnoses of neuroepithelial neoplasms. Cancer. 2000; 88(10):2342-9. [DOI:10.1002/(SICI)10970142(20000515)88:10<2342::AID-CNCR19>3.0.CO;2-X]

[11] Tugcu B, Kepoglu U, Gunal M, Gunaldi O, Karakaya B, Demirgil BT. Two distinct primary brain tumors, in same region of the same patient: A case report. Journal of NeuroOncology. 2006; 79(2):219-20. [DOI:10.1007/s11060-006-91304] [PMID]

[12] Suzuki K, Momota H, Tonooka A, Noguchi H, Yamamoto $\mathrm{K}$, Wanibuchi M, et al. Glioblastoma simultaneously present with adjacent meningioma: Case report and review of the literature. Journal of Neuro-Oncology. 2010; 99(1):147-53. [DOI:10.1007/s11060-009-0109-9] [PMID]

[13] Schwaighofer BW, Hesselink JR, Press GA, Wolf RL, Healy ME, Berthoty DP. Primary intracranial CNS lymphoma: MR manifestations. American Journal of Neuroradiology. 1989;10(4):725-9. [PMID]

[14] Widdel L, Kleinschmidt-DeMasters BK, Kindt G. Tumorto-tumor metastasis from hematopoietic neoplasms to meningiomas:Report of two patients with significant cerebral edema. World Neurosurgery. 2010; 74(1):165-71. [DOI:10.1016/j.wneu.2010.01.021] [PMID] 
This Page Intentionally Left Blank 